\title{
Fractional Fourier Transform Based Co-Radar Waveform: Experimental Validation
}

\author{
Domenico Gaglione*, Carmine Clemente*, Adriano Rosario Persico*, \\ Christos V. Ilioudis*, Ian K. Proudler ${ }^{\dagger}$, and John J. Soraghan* \\ *University of Strathclyde, CeSIP, EEE, 204, George Street, G1 1XW, Glasgow, UK \\ E-mail: domenico.gaglione, carmine.clemente, c.ilioudis, adriano.persico, j.soraghan@ @strath.ac.uk \\ $\dagger$ School of Electronic, Electrical and Systems Engineering, Loughborough University, Leicestershire, UK \\ E-mail: i.k.proudler@lboro.ac.uk
}

\begin{abstract}
A Fractional Fourier Transform (FrFT) based waveform design for joint radar-communication systems (CoRadar) that embeds data into chirp sub-carriers with different time-frequency rates has been recently presented. Simulations demonstrated the possibility to reach data rates as high as $3.660 \mathrm{Mb} / \mathrm{s}$ while maintaining good radar performance compared to a Linear Frequency Modulated (LFM) pulse that occupies the same bandwidth. In this paper the experimental validation of the concept is presented. The system is considered in its basic configuration, with a mono-static radar that generates the waveforms and performs basic radar tasks, and a communication receiver in charge of the pulse demodulation. The entire network is implemented on a Software Defined Radio (SDR) device. The system is then used to acquire data and assess radar and communication capabilities.
\end{abstract}

\section{INTRODUCTION}

The employment of joint radar-communication systems represent an innovative solution to the problem of continuously increasing demand on bandwidth [1] and the need to meet the low-SWaP (Size, Weight and Power consumption) requirements.

Applications that can benefit from the technology proposed in this paper include satellite and airborne Synthetic Aperture Radars (SARs) that need to share sensed data with a ground station rapidly [2]; nodes in a surveillance Multiple-Input Multiple-Output (MIMO) radar network that need to exchange information about targets; vehicles that need to interact in a intelligent transportation network [3]. All these applications may benefit from a joint-radar communication technology which shares bandwidth, power and hardware resources to perform radar and communication operations simultaneously.

A joint radar-communication system (Co-Radar) based on Fractional Fourier Transform (FrFT) waveform has been recently presented by the authors in [4]. The FrFT [5] was shown to be suitable for orthogonal waveforms generation for MIMO radar systems [6]-[9], and in this scenario it has been exploited to map complex modulated symbols into different chirp, or Linear Frequency Modulated (LFM), sub-carriers with different time-frequency rates. Simulations demonstrated the feasibility to reach data rate as high as $3.660 \mathrm{Mb} / \mathrm{s}$ at ranges up to $50 \mathrm{Km}$, while maintaining good radar performance in terms of range resolution, Doppler resolution and Side Lobe Levels (SLLs) when compared with a LFM pulse that occupies the same bandwidth.

Different techniques of embedding data in the radar waveform have been previously proposed. In [10] and [11] informa- tion bits are sent by exploiting the orthogonality between upchirp and down-chirp signals. Methods based on LFM pulses modulated through Binary Phase Shift Keying (BPSK) and Minimum Shift Keying (MSK) symbols are presented in [12] and [13], respectively. Stepped-frequency [14] and Frequency Modulated Continuous Wave (FMCW) [15] based joint radarcommunication systems have also been proposed, as well as Orthogonal Frequency Division Multiplexing (OFMD) based systems [16], [17]. However, none of these previous techniques is designed to achieve data rates up to $3.660 \mathrm{Mb} / \mathrm{s}$ at medium ranges.

In this paper the experimental validation of the FrFT CoRadar system [4] is reported. A basic configuration of the system is considered: a mono-static radar generates the FrFT waveform, sends the pulses, listens to echoes and performs basic radar tasks, while a communication receiver performs the pulse demodulation.

The remainder of the paper has the following structure. Section II summarises the concept of the FrFT based Co-Radar waveform design presented in [4]. Section III describes its implementation on a Software Defined Radio (SDR) device, while the experimental setup and results are presented in Section IV. Finally, Section V concludes the paper.

\section{FRFT BASED CO-RADAR SYSTEM}

In this section the FrFT based Co-Radar system [4] is presented. The FrFT [5] is a time-frequency representation of a signal and can be considered as a rotation by an arbitrary angle $\phi$ of the signal itself in the time-frequency plane, such that:

$$
\phi=\frac{\pi}{2} \alpha
$$

where $\alpha$ is called order of the transform. As mentioned earlier, FrFTs with different orders are used to map the modulated symbols into quasi-orthogonal chirp sub-carriers.

The block diagrams of the mono-static radar and the communication receiver in a simple FrFT Co-Radar system scenario are shown in Figure 1 and described in the following.

\section{A. Mono-Static Radar}

The block diagram of the mono-static radar in the considered basic configuration of the FrFT Co-Radar system is shown at the top of Figure 1. The serial-to-parallel (S/P) block is used to divide the long sequence of bits coming from the source into $C-1$ segments of $N$ bits each, where $C$ is the number of chirp 

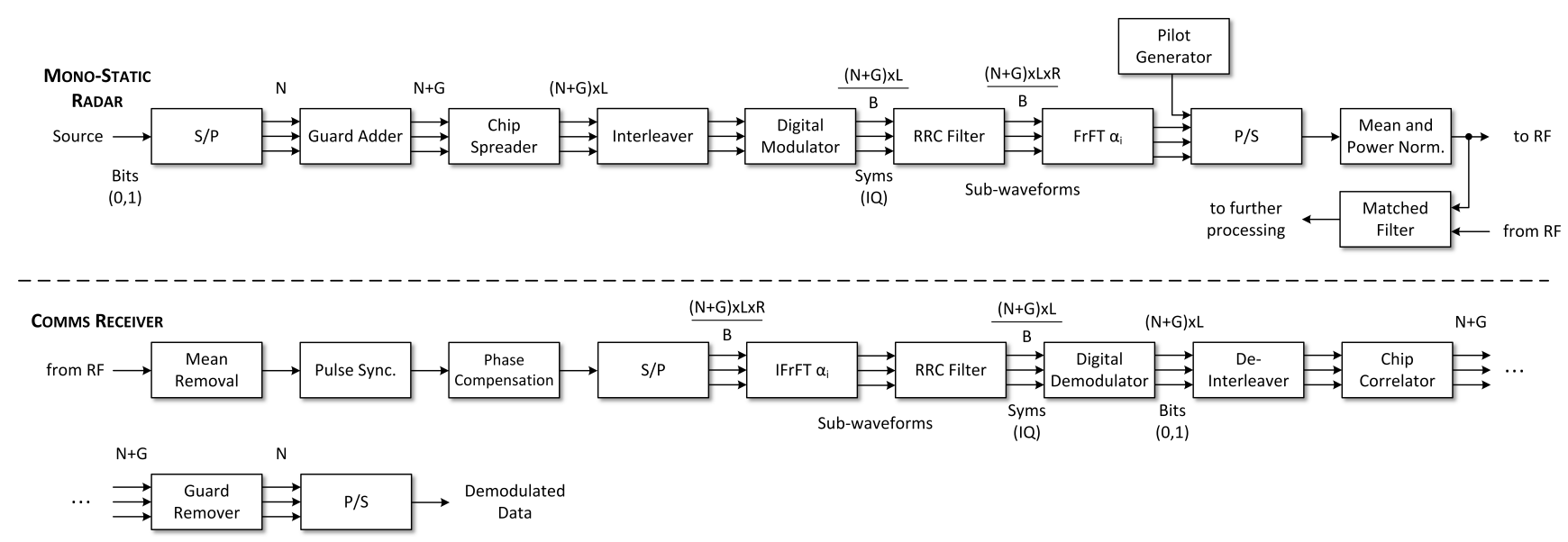

Figure 1. Block diagram of (top) the Mono-Static Radar and (bottom) the Communication Receiver of the FrFT based Co-Radar basic configuration [4].

sub-carriers that compose the waveform. The 0-th order subcarrier is not used to carry information bits. It accommodates a pilot waveform instead, used at the communication receiver for synchronisation and phase compensation. Since each pulse contains $N \times(C-1)$ information bits, the final bit rate is $N \times(C-1) \times \mathrm{PRF} b / \mathrm{s}$, where PRF stands for Pulse Repetition Frequency.

In each segment, $G$ guard bits are added at the end of the sequence in order to compensate for the group delay introduced by the Root Raised Cosine (RRC) filter. Then the sequence is spread by using a chip sequence; the chosen chip sequence is a $L$-long Barker code, which leads to a spread sequence of $(N+G) \times L$ bits.

The interleaver is used to mitigate the Inter-Carrier Interference (ICI) that occurs in the middle of the sequence due to the overlapping of the different chirp sub-carriers and that generates a burst of errors. It is applied only to the $N$ bits of information and aims to spread the burst of errors across the entire spread sequence.

The digital modulator maps a series of $B$ bits to one of the $M=2^{B}$ possible complex symbols belonging to the chosen modulation scheme (i.e. PSK), leading to a $(N+G) \times L / B$ long symbol sequence. The modulation scheme and the cardinality of its alphabet $M$ can be adaptively chosen according to the conditions of the channel.

The RRC filter is used to minimise the Inter-Symbol Interference (ISI) that may be caused by the channel. For efficiency, it is implemented as a multirate filter that upsamples the output by a factor $R$, leading to a final sequence of $(N+G) \times L \times R / B$ samples.

The $C-1$ sub-waveforms obtained after the RRC filter are then mapped to different chirp sub-carriers uniformly spaced in the time-frequency domain. Since the FrFT is periodic in $\alpha$ with period 4 , the uniformly spaced sub-carriers are obtained by choosing the $i$-th fractional order to be equal to $\alpha_{i}=i \bar{\alpha}, i=1, \ldots, C-1$, where $\bar{\alpha}=\frac{2}{C}$. Finally, the parallel-to-serial $(\mathrm{P} / \mathrm{S})$ block combines the chirp modulated sub-waveforms and the pilot sub-waveform by adding them together. Specifically, the latter is a bi-phase coded signal run by a Coarse/Acquisition (C/A) code [18] given by:

$$
p[n]=e^{j \pi\left(a[n]-\frac{1}{4}\right)}
$$

where $a[n]$ is the selected C/A code properly up-sampled and truncated to match the length, in samples, of the Co-Radar waveform.

Before sending the waveform to the Radio Frequency (RF) front-end, its mean is removed and the power is normalised such that all the transmitted pulses present the same power. While generating and transmitting the FrFT based pulses, the mono-static radar acquires the echoes and performs any needed radar task (i.e. matched filter with the transmitted pulse).

The spectrogram of a Co-Radar waveform with relatively few sub-carriers is shown in Figure 2 for clarity, although in practice many more sub-carriers would most likely be used. Each of them is the rotation by a specific angle, driven by the order of the FrFT, of a phase modulated signal (i.e. QPSK).

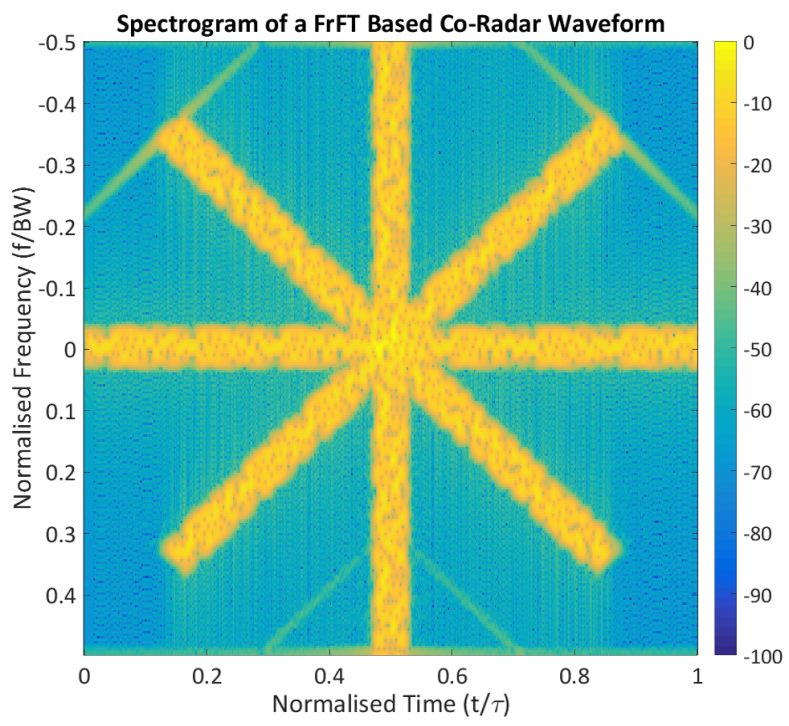

Figure 2. Spectrogram of a FrFT based Co-Radar waveform with 4 subcarriers.

\section{B. Communication Receiver}

The communication receiver is shown at the bottom of Figure 1. All the operations are performed within a Pulse Repetition Interval (PRI). Before the pulse is synchronised and demodulated, the mean of the received signal is subtracted to remove the strong return from the background. The synchronisation is necessary since the pulse could fall anywhere within the PRI due to the transmitter-receiver distance, and 


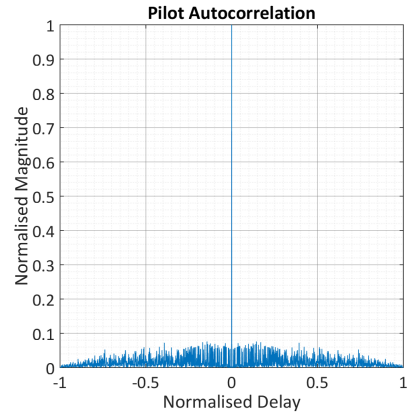

(a)

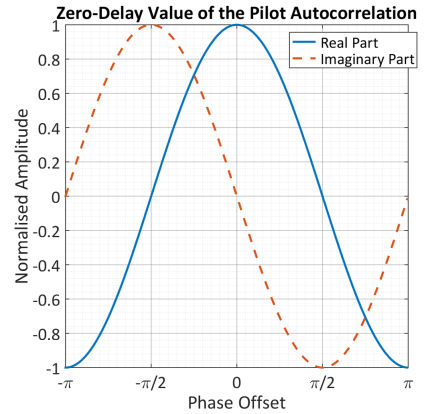

(b)
Figure 3. Pilot waveform (a) autocorrelation and (b) its zero-delay real and imaginary value on varying the phase offset.

the alignment with the pulse on a sample basis is needed to perform the inverse FrFT. This is achieved by exploiting the good autocorrelation properties of the pilot waveform, shown in Figure 3(a).

The pilot waveform is also used to estimate the phase offset introduced by the channel. Figure 3(b) shows how the complex zero-delay value of the pilot waveform autocorrelation changes with varying the phase offset. In particular, when the offset is zero the real part of the zero-delay value reaches its maximum, while the imaginary part is zero. Hence, once the peak of the autocorrelation is detected by matched filtering the received signal with the pilot waveform, the phase offset can be estimated by evaluating the phase of the correlation peak. This phase estimation method is also robust with respect to the Doppler shift potentially present in the signal.

Once the synchronisation and the phase compensation are performed, the pulse can be demodulated. The S/P block splits and redirects the pulse, whose length is $(N+G) \times L \times R / B$ samples, to $C-1$ different IFrFT blocks that perform the inverse FrFT. Each sequence is then input of the RRC filter, which also down-samples the sub-waveform by a factor $R$. The digital demodulator translates the $(N+G) \times L / B$ long sequence of symbols in a sequence of $(N+G) \times L$ bits, according to the modulation employed. At this point, the deinterleaver performs the inverse operation of the interleaver.

The chip correlator block correlates the input spread sequence with the $L$-long Barker chip code used in transmission to extract $N+G$ bits, exploiting both the low correlation side lobes and the knowledge that the peaks of the correlation occur every $L$ samples. Finally, the guard remover and the P/S blocks reconstruct the original stream by combining the $N$-long bit sequences coming from the $C-1$ different parallel branches.

\section{FRFT CO-RADAR ON SDR}

The FrFT Co-Radar system is implemented by means of a SDR device, namely the National Instruments Universal Software Radio Peripheral (NI-USRP) 2943r. It has four inphase and quadrature (IQ) channels, two receivers and two transmitters/receivers, and its working frequency ranges between $1.2 \mathrm{GHz}$ and $6.6 \mathrm{GHz}$. It is provided with a fully programmable Xilinx Kintex-7 FPGA (Field-Programmable Gate Array) and can be connected to a host computer through a high-speed, low-latency PCI Express x4 ( 800 MB/s) connector. It is used with three wideband LB-2678-15 multi octave horn antennas produced by A-Info, two for the mono-static radar node and one for the communication receiver.

The USRP $2943 r$ is programmed through NI software

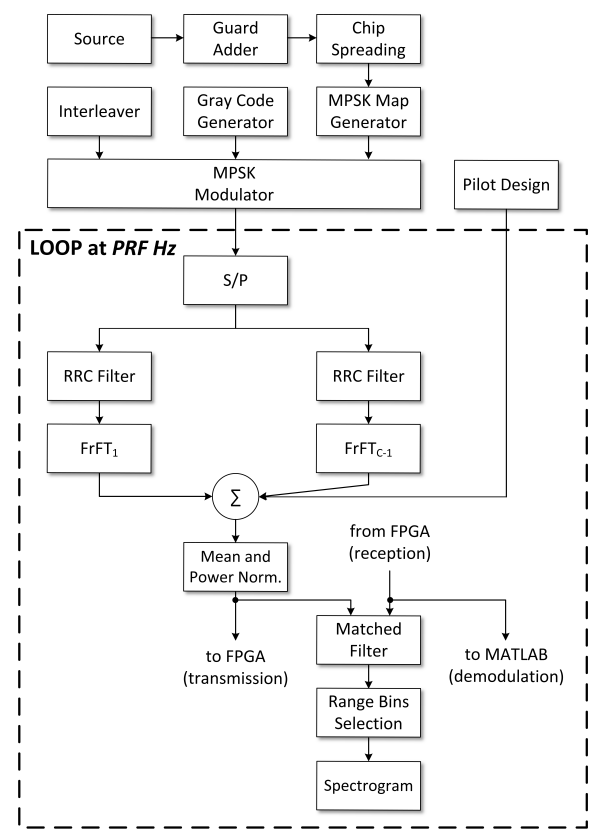

Figure 4. High-level block diagram of the FrFT Co-Radar system implementation in LabVIEW.

LabVIEW, and its high-level block diagram is shown in Figure 4. Within the loop that repeats every PRI, the FrFT Co-Radar waveform is generated as described in the previous section and sent to the FPGA. The latter up-samples the signal in transmission to meet the $120 \mathrm{MHz}$ data clock frequency of the device and interfaces with the RF front-end. Meanwhile, the received signal, down-sampled and coming from the FPGA, is sent both to a MATLAB session for the real-time pulse demodulation and to a matched filter with the transmitted pulse for further radar processing. Specifically, a real-time spectrogram is computed. This choice is driven by the limited power and bandwidth of the employed SDR device, which are not sufficient for more advanced radar operations. Outside the loop, the message to send is loaded and all the preliminary steps are performed.

\section{EXPERIMENTAL SETUP AND RESUlTS}

The implemented FrFT Co-Radar system is used in a controlled laboratory environment to acquire data and assess its communication and radar capability. The acquisition geometry is shown in Figure 5. The mono-static radar is placed at the

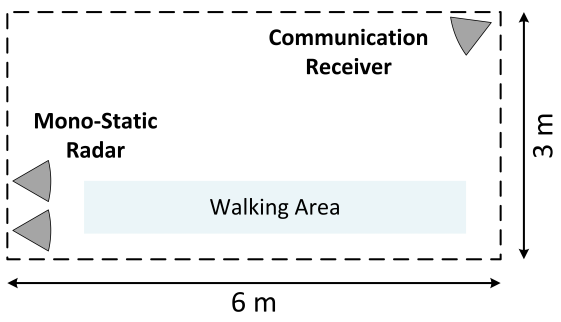

Figure 5. Acquisition geometry of the laboratory-based experimental campaign.

bottom left, the communication receiver at the top right, while within the light blue area a person is walking towards and away from the radar to generate a Doppler signal.

The transmitted message is a $64 \times 64$ black and white 
image with a bit depth 5. Carrier frequency is $3 \mathrm{GHz}$ and the bandwidth is $1 \mathrm{MHz}$. The number of information bits per carrier, $N$, is 3 , the length of the Barker code is $L=7$ and Quadrature PSK (QPSK) is the employed modulation scheme, hence $B=2$. The RRC filter is designed to span $S=8$ symbols, with an up-sampling factor $R=18$ and a roll-off factor $\beta=0.4$. This leads to a guard of $G=3$ bits. The total number of samples per waveform is 378 , which means that the duration of the pulse is $\tau=378 \mu \mathrm{s}$. The PRF is fixed to $83.33 \mathrm{~Hz}$, giving a duty cycle of $3.15 \%$.

Different configurations are analysed by changing the number of sub-carriers, $C=4,6,8,10$, and modifying the transmitted power. The Signal-to-Noise Ratio (SNR) is estimated both at the mono-static radar and the communication receiver.

Communication performance is shown with solid lines in Figure 6, in terms of Bit Error Ratio (BER) averaged over 10 realisations vs $S N R_{\text {comms }}$. The dotted lines in Figure 6 show

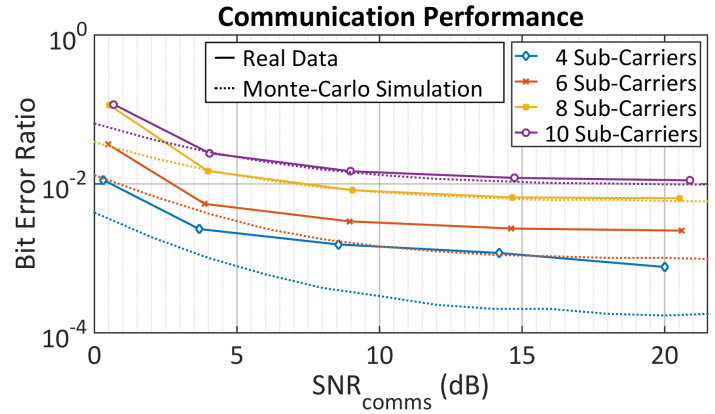

Figure 6. Communication performance on varying the $S N R_{\text {comms }}$ and for different number of chirp sub-carriers.

the results obtained by running Monte-Carlo simulations with $10^{5}$ iterations with the same parameters listed above, assuming the channel to be Rician with $K$-factor equal to $6 \mathrm{~dB}$ (indoor channel). They are used as comparison to validate the results on the acquired data. Note that no Forward Error Correction (FEC) method is used, since this is out of the scope of the present paper.

As expected, as the $S N R_{\text {comms }}$ increases, the BER decreases. However, no significant further improvements are observed for $S N R_{\text {comms }}$ greater than $15 \mathrm{~dB}$, and this trend is confirmed by the results from the Monte-Carlo simulations, which clearly show plateaus. It is observed that superior results are obtained when 4 and 6 sub-carriers are used, and this can be explained by the higher ICI that waveforms with 8 and 10 sub-carriers experience.

The radar capabilities of the FrFT Co-Radar are presented by showing spectrograms from the signals acquired during the laboratory-based experimental campaign. Figure 7 and Figure 8 show spectrograms when FrFT Co-Radar pulses with $C=4$ and $C=8$ sub-carriers are used, respectively, and for two different values of $S N R_{\text {radar. }}$ In all the cases the Doppler and micro-Doppler signature of the person walking towards and away from the radar is clearly visible.

\section{CONCLusion}

The paper presented an experimental validation of the FrFT based Co-Radar system proposed in [4]. The system was successfully implemented on a SDR device and its performance demonstrated in a controlled laboratory environment. A basic configuration was considered with one mono-static radar and

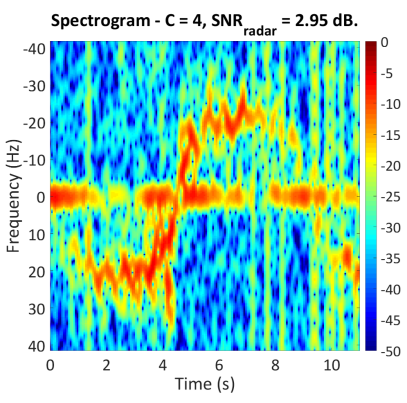

(a)

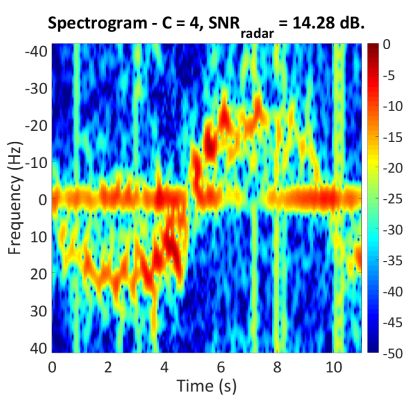

(b)
Figure 7. Spectrograms obtained from FrFT Co-Radar pulses with $C=4$ and different $S N R_{\text {radar }}$. Window length 0.36 seconds, overlap $80 \%$. Person walking towards the radar approximately between $0-5$ seconds and 10-11 seconds, and away from it between 5-10 seconds.

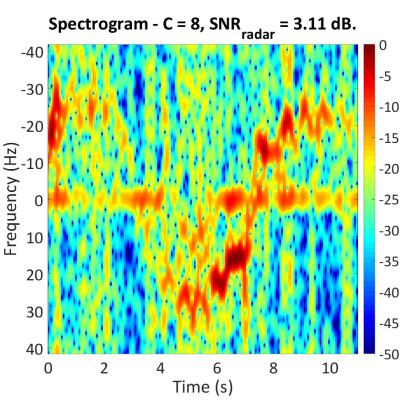

(a)

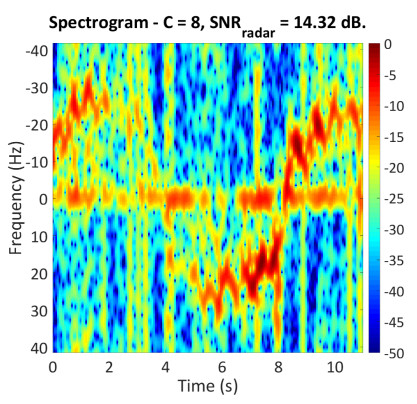

(b)
Figure 8. Spectrograms obtained from FrFT Co-Radar pulses with $C=8$ and different $S N R_{\text {radar }}$. Window length 0.36 seconds, overlap $80 \%$. Person walking towards the radar approximately between 4-8 seconds, and away from it between $0-4$ seconds and 8-11 seconds.

one communication receiver.

Communication performance was evaluated in terms of BER vs $S N R_{\text {comms }}$ and resulted consistent with that obtained from Monte-Carlo simulations. It shows BER between $10^{-3}$ and $10^{-2}$ when no error detection and correction techniques are used. Due to the limited power and bandwidth of the employed SDR device, the radar capability of these novel waveforms are assessed by computing the spectrograms of the acquired signals. They clearly showed the main Doppler and micro-Doppler signature of a person walking towards and away from the radar. These preliminary results confirm the capability of the proposed novel waveforms of joint radar-communication operations.

\section{ACKNOWLEDGMENT}

This work was supported by the Engineering and Physical Sciences Research Council (EPSRC) Grant number EP/K014307/1 and the MOD University Defence Research Collaboration in Signal Processing.

\section{REFERENCES}

[1] H. Griffiths, "The Challenge of Spectrum Engineering," in 2014 11th European Radar Conference (EuRAD), Oct 2014, pp. 1-4.

[2] S. Quan, W. Qian, J. Guq, and V. Zhang, "Radar-Communication Integration: An Overview," in 2014 IEEE 7th International Conference on Advanced Infocomm Technology (ICAIT), Nov 2014, pp. 98-103.

[3] D. Erricolo, H. Griffiths, L. Teng, M. C. Wicks, and L. L. Monte, "On the Spectrum Sharing Between Radar and Communication Systems," in 2014 International Conference on Electromagnetics in Advanced Applications (ICEAA), Aug 2014, pp. 890-893. 
[4] D. Gaglione, C. Clemente, C. V. Ilioudis, A. R. Persico, I. Proudler, and J. J. Soraghan, "Fractional Fourier Based Waveform for a Joint RadarCommunication System," in 2016 IEEE Radar Conference (RadarConf), Philadelphia, USA, May 2016, pp. 811-816.

[5] H. M. Ozaktas, Z. Zalevsky, and M. A. Kutay, The Fractional Fourier Transform with Applications in Optics and Signal Processing. Wiley, Chichester, 2001.

[6] C. Clemente, I. Shorokhov, I. Proudler, and J. Soraghan, "Radar Waveform Libraries Using Fractional Fourier Transform," in 2014 IEEE Radar Conference, May 2014, pp. 0855-0858.

[7] C. Clemente, C. Ilioudis, D. Gaglione, K. Thompson, S. Weiss, I. Proudler, and J. Soraghan, "Reuse of Fractional Waveform Libraries for MIMO Radar and Electronic Countermeasures," in 2014 6th International Symposium on Communications, Control and Signal Processing (ISCCSP), May 2014, pp. 505-508.

[8] C. Ilioudis, C. Clemente, I. Proudler, and J. Soraghan, "Constant Envelope Fractional Fourier Transform Based Waveform Libraries for MIMO Radar," in 2014 Sensor Signal Processing for Defence (SSPD), Sept 2014, pp. 1-5.

[9] C. Ilioudis, C. Clemente, I. Proudler, and J. Soraghan, "Performance Analysis of Fractional Waveform Libraries in MIMO Radar Scenario," in 2015 IEEE Radar Conference (RadarCon), May 2015, pp. 11191124.

[10] M. Roberton and E. Brown, "Integrated Radar and Communications Based on Chirped Spread-Spectrum Techniques," in 2003 IEEE MTTS International Microwave Symposium Digest, vol. 1, June 2003, pp. 611-614.

[11] G. N. Saddik, R. Singh, and E. Brown, "Ultra-Wideband Multifunctional Communications/Radar System," IEEE Transactions on Microwave Theory and Techniques, vol. 55, no. 7, pp. 1431-1437, July 2007.

[12] Z. Zhao and D. Jiang, "A Novel Integrated Radar and Communication Waveform Based on LFM Signal," in 2015 5th International Conference on Electronics Information and Emergency Communication (ICEIEC), May 2015, pp. 219-223.

[13] X. Chen, X. Wang, S. Xu, and J. Zhang, "A Novel Radar Waveform Compatible with Communication," in 2011 International Conference on Computational Problem-Solving (ICCP), Oct 2011, pp. 177-181.

[14] F. Hu, G. Cui, W. Ye, L. Kong, Y. Huang, and L. Yuan, "Integrated Radar and Communication System Based on Stepped Frequency Continuous Waveform," in 2015 IEEE Radar Conference (RadarCon), May 2015, pp. 1084-1087.

[15] W. Scheiblhofer, R. Feger, A. Haderer, and A. Stelzer, "Method to Embed a Data-Link on FMCW Chirps for Communication Between Cooperative 77-GHz Radar Stations, year $=2015$, pages $=181-184$, month = Sept,", in Radar Conference (EuRAD), 2015 European.

[16] C. Sturm and W. Wiesbeck, "Waveform Design and Signal Processing Aspects for Fusion of Wireless Communications and Radar Sensing," Proceedings of the IEEE, vol. 99, no. 7, pp. 1236-1259, July 2011.

[17] J. Zhao, K. Huo, and X. Li, "A Chaos-Based Phase-Coded OFDM Signal for Joint Radar-Communication Systems," in 2014 12th International Conference on Signal Processing (ICSP), Oct 2014, pp. 19972002.

[18] GPS ICD, "Global Positioning Systems Directorate System Engineering \& Integration Interface Specification IS-GPS-200H," Navstar GPS Space Segment/Navigation User Interfaces, 2013. 